\title{
Application of Excitation from Multiple Locations on a Simplified High-Lift System
}

\author{
LaTunia Pack Melton* and Chung-Sheng Yao ${ }^{\dagger}$ \\ Flow Physics and Control Branch \\ NASA Langley Research Center, Hampton, VA 23681 \\ Avi Seifert $\ddagger$ \\ Tel-Aviv University, Ramat-Aviv 69978, ISRAEL
}

\begin{abstract}
A series of active flow control experiments were recently conducted on a simplified highlift system. The purpose of the experiments was to explore the prospects of eliminating all but simply hinged leading and trailing edge flaps, while controlling separation on the supercritical airfoil using multiple periodic excitation slots. Excitation was provided by three, independently controlled, self-contained, piezoelectric actuators. Low frequency excitation was generated through amplitude modulation of the high frequency carrier wave, the actuators' resonant frequencies. It was demonstrated, for the first time, that pulsed modulated signal from two neighboring slots interact favorably to increase lift. Phase sensitivity at the low frequency was measured, even though the excitation was synthesized from the high-frequency carrier wave. The measurements were performed at low Reynolds numbers and included mean and unsteady surface pressures, surface hot-films, wake pressures and particle image velocimetry. A modest $(6 \%)$ increase in maximum lift (compared to the optimal baseline) was obtained due to the activation of two of the three actuators.
\end{abstract}

\section{Nomenclature}

$c \quad$ airfoil chord (at $\delta_{s}=\delta_{f}=0^{\circ}$ )

$C_{\mu} \quad$ excitation momentum coefficient, $\equiv\left\langle J^{\prime}\right\rangle / c q$

$C_{d p} \quad$ pressure drag coefficient

$C_{D} \quad$ total drag

$C_{L} \quad$ lift coefficient

$C_{L, \text { max }}$ maximum lift coefficient

$C_{p} \quad$ pressure coefficient, $\equiv\left(P-P_{s}\right) / q$

$C_{p, \min }$ minimum pressure coefficient

Dcy duty cycle

$f \quad$ oscillation frequency, $\mathrm{Hz}$

$F^{+} \quad$ reduced frequency, $\equiv\left(f x_{s p}\right) / U_{\text {inf }}$

$h \quad$ slot width

$J^{\prime} \quad$ oscillatory momentum at slot exit, $\equiv \rho h u_{j}^{\prime 2}$

$N \quad$ Number of cycles of the modulating waveform

$P \quad$ pressure

$P_{s} \quad$ static pressure

\footnotetext{
* Member of AIAA, latunia.p.melton@nasa.gov

†c.s.yao@nasa.gov

¥Associate Fellow, AIAA, seifert@eng.tau.ac.il, Senior lecturer, Dept. of Fluid Mechanics and Heat Transfer, School of Mechanical Eng., Faculty of Engineering. Also, visiting scientist, National Institute of Aerospace, Hampton, VA.

Copyright (C) 2004 by the American Institute of Aeronautics and Astronautics, Inc. The U.S. Government has a royalty-free license to exercise all rights under the copyright claimed herein for Governmental purposes. All other rights are reserved by the copyright owner.
} 
$q \quad$ freestream dynamic pressure, $\equiv 1 / 2 \rho U_{\infty}^{2}$

$R e_{c} \quad$ chord Reynolds number, $\equiv U_{\infty} c / \nu$

$x_{s p} \quad$ distance from actuator to trailing edge

$U, u \quad$ average and fluctuating streamwise velocity

$x / c \quad$ normalized streamwise location

$z \quad$ spanwise location

$\alpha \quad$ angle of attack

$\delta_{f} \quad$ TE flap deflection

$\delta_{s} \quad$ LE flap deflection

$\nu \quad$ kinematic viscosity

$\rho$ density

\section{A. Abbreviations}

$2 D$ two-dimensional

$A F C \quad$ active flow control

$A M \quad$ amplitude modulation

$B L \quad$ boundary layer

$B M \quad$ Burst Modulation

$L E \quad$ leading edge

$T E \quad$ trailing edge

$V S F \quad$ vortex shedding frequency

\section{B. Subscripts}

$\begin{array}{ll}\mathrm{j} & \text { conditions at excitation slot } \\ \mathrm{m} & \text { modulation } \\ \mathrm{r} & \text { resonance } \\ \mathrm{S} & \text { separation } \\ \infty & \text { freestream conditions }\end{array}$

\section{Superscript \\ root mean square of fluctuating value}

\section{Introduction}

Numerous experiments at both low $^{1}$ and high ${ }^{2,3}$ Reynolds numbers have shown that periodic excitation is effective as well as efficient in terms of momentum at controlling separation. This information combined with that of a system study, ${ }^{4}$ indicating the possibility of significant payoffs such as net airplane cost, weight, and cruise drag reductions has led to the application of active separation control to a simplified high-lift system. The purpose of this study is to explore ways to simplify current multi-element high-lift systems and airfoils ${ }^{5}$ that use slots and the Fowler effect to generate high lift. The chosen design completely eliminates hinges and positioning actuators that need to be external to the airfoil contour as well as passive slots for energizing the boundary layer. All hinges and positioning actuators in the proposed configuration will be internal, and thus reduce parasitic drag at cruise. The leading edge (LE) flap is used to increase $C_{L, \max }$ due to increased circulation and prevention of laminar leading edge separation at high incidence. Zero-mass-flux periodic excitation, directed downstream at a shallow angle to the local surface, is applied at locations that are prone to separation, i.e. the LE and trailing edge (TE) flap shoulders.

Flow control research using steady momentum transfer on a high-lift system dates back to the 1930 's. ${ }^{6}$ Additional interest was spurred in the 1950's by the use of the gas turbine engine. The research showed that separation could be controlled effectively using wall-tangential steady momentum transfer but that the momentum requirement was very large. ${ }^{6}$ The use of periodic excitation for separation control on the simply hinged high-lift system should reduce the momentum requirements compared to that of steady excitation 
based on results from experiments on other airfoils. ${ }^{2,3}$ In addition, research using pulsed excitation has also shown that the momentum requirements can be reduced further by varying the duty cycle of the excitation. ${ }^{7}$

The results obtained when applying periodic excitation at the LE flap shoulder of this airfoil were reported in a previous publication. ${ }^{8}$ High frequency periodic excitation, typical of the piezoelectric actuators currently used, was applied at the LE flap shoulder, and delayed stall and increased $C_{L, \max }$ by $10-15 \%$, at low TE flap deflections. It was shown that low frequency amplitude modulation could be used to achieve similar benefits in aerodynamic performance and required $50 \%-70 \%$ less $C_{\mu}$.

The effect of applying excitation at various locations along the trailing edge flap was also examined. ${ }^{9}$ It was found that the optimal flap deflection for an excitation slot location was very sensitive to changes in the surface and this was attributed to surface curvature. Amplitude modulation of the high frequency excitation reduced the momentum requirements by a factor of two to three. The momentum requirements for controlling separation on the trailing edge flap were significantly higher than those required for control of separation near the leading edge flap shoulder and also as compared to high Reynolds number experiments performed on a NACA $0015 .^{2}$

To increase the momentum available for controlling separation on the TE flap, we examine the effect of combining the excitation from an actuator just upstream of the trailing edge flap (i.e., on the most aft region of the main element) with the excitation from an actuator just downstream of the trailing edge flap shoulder (i.e. on the forward part of the TE flap). The effects of phase angle and duty cycle, Dcy, are presented as well. The effect of combining the TE flap excitation with the excitation emanating from the LE flap actuator is also presented and discussed.

\section{Experiment}

The experiments were conducted in the Basic Aerodynamics Research Tunnel (BART) at the NASA Langley Research Center. Details about the wind tunnel and instrumentation can be found in Ref. 8 . Included here are details about the model and actuator used for controlling flow separation on the TE flap.

\section{A. 2D Simplified High-Lift Model}

The simplified high-lift version of the NASA EET airfoil ${ }^{5}$ was designed in a modular manner so that zero-netmass-flux actuators could replace solid regions in the model near the LE and TE flap shoulders (Fig. 1(a)). The $406.4 \mathrm{~mm}$ chord model has a $15 \%$ chord LE flap that can be deflected from $0^{\circ}$ to $-30^{\circ}$ and a $25 \%$ chord trailing edge flap that can be deflected from $0^{\circ}$ to $60^{\circ}$. Angle of attack settings for the airfoil and the two flaps were automated and closed-loop computer controlled. The model has 78 streamwise static pressure taps located at mid span and two rows of 18 spanwise static pressure taps, spaced $50.8 \mathrm{~mm}$ apart, located at $\mathrm{x} / \mathrm{c}=0.35$ and $\mathrm{x} / \mathrm{c}=0.94$ on the upper surface. In addition to the static pressure taps, there are nine unsteady pressure transducers on the model surface and at least one unsteady pressure transducer embedded in each actuator cavity for monitoring the pressure fluctuations produced by the actuator and correlating the wind tunnel experiment with the bench-top actuator calibration tests (in order to evaluate $C_{\mu}$ ).

\section{B. Flap Actuator}

An internal piezoelectric actuator was used on the TE flap (Figs. 1). The flap actuator, with its four alternative excitation slots, all inclined at about $30^{\circ}$ to the surface and facing downstream, is shown in Fig. 1(b). The three upstream slots are $0.635 \mathrm{~mm}$ wide, and the aft slot is $0.51 \mathrm{~mm}$ wide. The $\mathrm{x} / \mathrm{c}$ locations and slot widths for the flap actuator slots $\left(\delta_{f}=0^{\circ}\right)$ are given in Table 1.

Table 1. Flap actuator slot locations

\begin{tabular}{ccc}
\hline Slot & $\mathrm{x} / \mathrm{c}$ location at $\delta_{f}=0^{\circ}$ & slot width $(\mathrm{mm})$ \\
\hline FWD & 0.725 & 0.635 \\
$\# 3$ & 0.757 & 0.635 \\
Not Used & 0.790 & 0.635 \\
AFT & 0.845 & 0.51 \\
\hline
\end{tabular}




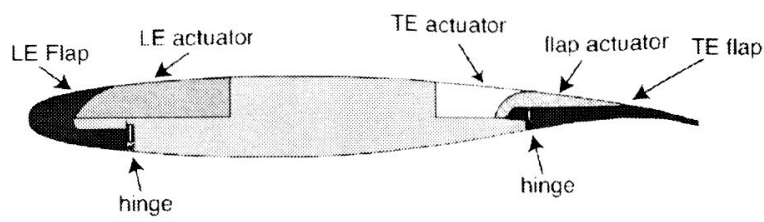

(a) EET airfoil with actuator regions indicated $\left(\delta_{f}=\delta_{s}=0^{\circ}\right)$.

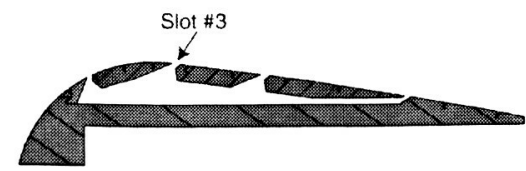

(b) Flap actuator cross--section showing alternative slot locations.

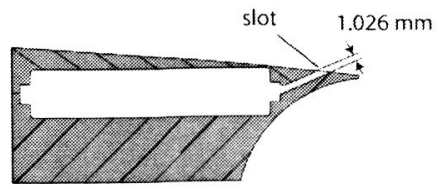

(c) TE actuator cross-section, TE slot $x / c=0.73$.

Figure 1. Modified EET model

The three forward slots each have 19 spanwise segments that are $0.051 \mathrm{~m}$ in length while the aft slot is continuous. A comprehensive bench-top calibration, using a single hot-wire that was traversed along the span of each slot with all other slots sealed, was performed on the flap actuator prior to installation in the tunnel. The unsteady pressures were measured in the actuator's cavity to monitor its operation during the calibration and while in the tumnel. The flap actuator was operated at its resonant frequency $(1 \mathrm{kHz} \pm 0.3$ $\mathrm{kHz}$, depending on the slot used) using a pure sine wave input signal and also with an amplitude modulation (AM) input signal at frequencies lower by an order of magnitude than the actuator's resonant frequency: Only one slot was active during each experiment. The three forward slots were sealed, when not in use, using a water-soluble filler to minimize surface discontinuities, and $0.051 \mathrm{~mm}$ thick, $12.7 \mathrm{~mm}$ wide kapton tape was used to seal the aft slot when not in use.

\section{TE Actuator}

A piezoelectric actuator with a resonant frequency of $1.3 \mathrm{kHz}$ was used on the TE region of the model upstream of the trailing edge flap, as shown in Figs. 1(a) and 1(c). The TE actuator's slot width is 1.026 $\mathrm{mm}$ and it is located at $\mathrm{x} / \mathrm{c}=0.73$.

\section{PIV Set-Up}

Two-dimensional digital particle image velocimetry (PIV) was used to measure the instantaneous flow fields, phase synchronized with the flap actuator cycle. The PIV system includes two $1.3 \mathrm{~K}$ x $1 \mathrm{~K}$ cameras installed side-by-side with $105 \mathrm{~mm}$ Macro lens. The fields of view of the two cameras were overlapped to capture the $\mathrm{TE}$ region of the model from slightly upstream of the TE actuator slot to at least the TE of the model. A small region of the surface of the model, in the overlap region, is lost because of camera spacing restrictions due to the lens and mounting system used. The size of the measurement plane was about $185 \mathrm{~mm}$ long by 96 $\mathrm{mm}$ high. A rectangular grid was used with a minimum resolution of $24 \times 24$ pixels. The maximum overlap between adjacent interrogation regions was $50 \%$. Two theatrical smoke generators were used for seeding the entire lab to assure uniform seeding. Dual Nd-Yag lasers (250 - $300 \mathrm{mj}$ per pulse) were used to illuminate a light sheet, placed about $50 \mathrm{~mm}$ to the left of the model centerline.

\section{E. Experimental Uncertainty}

The following uncertainty levels are provided for the data presented in this paper. The airfoil incidence angles, $\alpha$ 's, presented are accurate to within $\pm 0.03^{\circ}$. The LE and TE flap deflection angles are accurate to within $\pm 0.25^{\circ}, C_{\mu}$ is accurate to within $20 \%$ (partly due to slot width uncertainty of $\pm 0.08 \mathrm{~mm}$ and partly due to calibration uncertainties such as wire location and $\pm 2 \%$ uncertainty in hot-wire velocity measurements), and $R e_{c}$ is accurate to within $3 \%$. The uncertainties of the airfoil integral parameters are listed in Table 2 (in absolute values and related to flow conditions). 
Table 2. Uncertainty of Airfoil integral parameters

\begin{tabular}{cccc}
\hline \hline Parameter & Fully attached & Stalled & Controlled \\
\hline$C_{L}$ & 0.01 & 0.04 & 0.02 \\
$C_{d p}$ & 0.002 & 0.004 & 0.003 \\
$C_{D}$ & 0.002 & 0.008 & 0.006 \\
\hline \hline
\end{tabular}

The large uncertainty in the total drag, $C_{D}$, is due to the extrapolation of the wake data for some of the high-lift configurations of the airfoil, to wind tunnel interference, and to uncertainty about wind tunnel static pressure and wake rake location. It should be noted that the integral parameters in this paper were not corrected for the significant tunnel wall interference present in the BART facility for the model size used; however, the relative improvements due to actuation can be conservatively estimated without wall corrections.

\section{F. Test Conditions (flow and geometry)}

Most of the experiments using the TE flap actuators were conducted at incompressible values of $R e_{c}$ ranging from $0.24 \times 10^{6}$ to $0.75 \times 10^{6}$. The trailing edge flap deflection was varied from $\delta_{f}=0^{\circ}$ to $60^{\circ}$, and $\delta_{s}$ was between $0^{\circ}$ and $-30^{\circ}$.

\section{Results}

\section{A. TE Actuator Results}

The effects of the LE and flap excitations were discussed in References 8 and 9, respectively. Before discussing methods of combining these excitations with that of the TE actuator, the effect of the TE actuator on the flow around the simplified high-lift system is examined.

A significant improvement in lift, when introducing excitation from one of the flap actuator slots, was obtained when using flap slot \#3 with $\delta_{f}=20^{\circ}, \delta_{s}=-25^{\circ}$, and $\alpha=6^{\circ}$. Figure 2(a) shows $C_{L}$ versus TE flap deflection, $\delta_{f}$, for the TE actuator and the flap actuator (using slot \#3). A pure sine excitation $\left(F^{+}=11.9, C_{\mu} \approx 1.0 \%\right)$ emanates from the flap actuator and an AM excitation $\left(F^{+}=15.9, F_{M}^{+}=0.4\right.$, $C_{\mu} \approx 1.2 \%$ ) is provided by the $\mathrm{TE}$ actuator. The data indicate that the maximum improvement in lift occurs when the TE flap deflection is between $20^{\circ}$ to $25^{\circ}$ for both the TE and flap actuators operating alone. The reason for the effectiveness of the flap excitation is that separation occurs just downstream of slot \#3, however for the TE actuator the separation location is further downstream from the excitation slot location. Additional research is needed to determine the reason for the optimal flap deflection for control being similar for the TE and flap actuators. For the purpose of this study, it is beneficial for the two actuators to give the maximum improvement in lift at the same TE flap deflection because the goal is to improve airfoil performance by combining multiple excitation sources. The data shown in Fig. 2(a) indicate that combining the two excitations increases the lift by adding the effects. Furthermore, while the effect of each individual actuator nullifies at $\delta_{f} \approx 35^{\circ}$, the combined effect persists to at least $\delta_{f}=40^{\circ}$ and probably beyond. This point will be explored further in Section C. Figure 2(b) compares the pressure distributions for the data of Fig. 2(a) at $\delta_{f}=20^{\circ}$. The flap excitation creates a strong suction peak at the flap shoulder and a more positive pressure downstream of the slot while the AM excitation of the TE actuator mainly has an effect upstream of the TE slot of decreasing the pressure when compared to the baseline $C_{p}$. It seems that also in terms of the $C_{p}$ alteration, the effects of the two slot excitations add up.

The effectiveness of the three types of excitation signals; Pure Sine (PS), Amplitude Modulation (AM), and Burst Mode (BM), used during the experiment, are compared for the TE actuator. Figure 3 presents $C_{L}$ and $C_{d p}$ plotted against $C_{\mu}$. The data indicate, as was shown for excitations from the LE and TE actuators, ${ }^{8,9}$ that for a given increment in lift the momentum requirements are reduced when using AM excitation. The data also indicate that using BM can reduce the momentum requirements even further for $C_{\mu}>0.2 \%$. The momentum required to increase lift when excitation is introduced from the TE actuator is much larger than the momentum required when using slot\#3 of the flap actuator. A notable increase in lift 


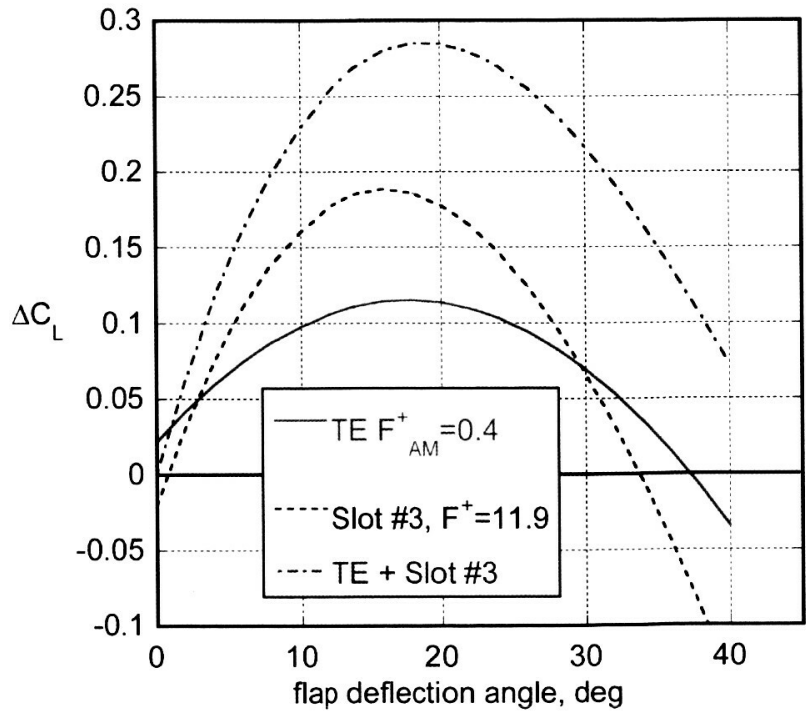

(a) $\Delta C_{L}$ vs flap deflection angle. Note the data was generated from curve fits of lift data

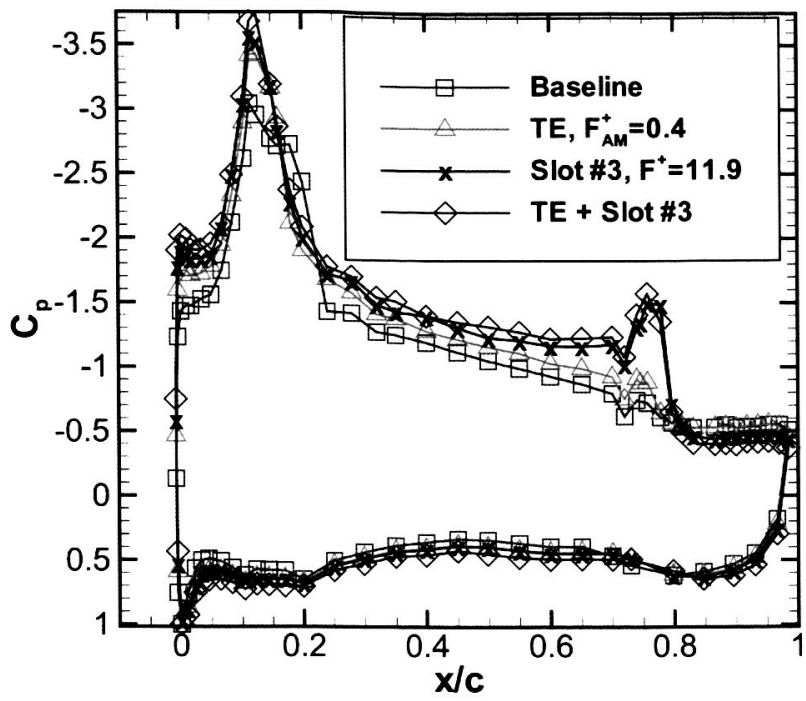

(b) Comparison of $C_{p}$ with control from flap actuator and TE actuator, $\delta_{f}=20^{\circ}$.

Figure 2. TE flap control. $R e_{c}=0.24 \times 10^{6}, \delta_{s}=-25^{\circ}, \alpha=6^{\circ}$.

requires $C_{\mu}>1.2 \%$ and $0.6 \%$ for the PS and AM excitations, respectively. For TE actuator $C_{\mu}$ levels below $1.2 \%$ with a PS excitation, $C_{L}$ initially increases, then decreases before finally increasing above the baseline $C_{L}$ level. When excitation was introduced from slot\#3 of the flap actuator there was a linear relationship between $C_{\mu}$ and lift. ${ }^{9}$ The increase in form-drag seen when applying AM excitation from flap slot \#3 (Ref. 9) is not observed when using the TE actuator.

\section{B. Burst Mode for the Flap and TE Actuators}

A major goal of the current research is to determine if improvements in airfoil performance can be obtained by combining multiple actuators, e.g., the TE and flap actuators. An additional goal is to determine if the excitation duty cycle can be reduced below the 0.50 Dcy of the AM excitation (i.e. pulsed excitation). In this section we examine the effect of Dcy on each actuator when operated individually.

Figure 4(a) presents data acquired when using burst mode (BM) with excitation emanating from flap actuator slot $\# 3$. The airfoil and flow conditions are $\alpha=6^{\circ}, R e_{c}=0.24 \times 10^{6}, \delta_{s}=-25^{\circ}$ and $\delta_{f}=20^{\circ}$. The data indicate that for a $D c y>0.45$ there are no further improvements in $C_{L}$, indicating that the additional power required to generate $D c y>0.45$ is wasted. The form-drag, $C_{d p}$, initially increases, reaches the baseline $C_{d p}$ value near a Dcy of 0.5, but never decreases below the baseline $C_{d p}$ value. This is an inherent feature of the low frequency excitation as compared to the high frequency pure sine excitation. This is probably associated with the vortex shedding process (Naim et $\mathrm{al}^{10}$ ) that "locks-in" with the excitation at this $F_{M}^{+}$. Also shown on Fig. 4(a) are $C_{L}$ and $C_{d p}$ generated by amplitude modulation, AM, with a square wave

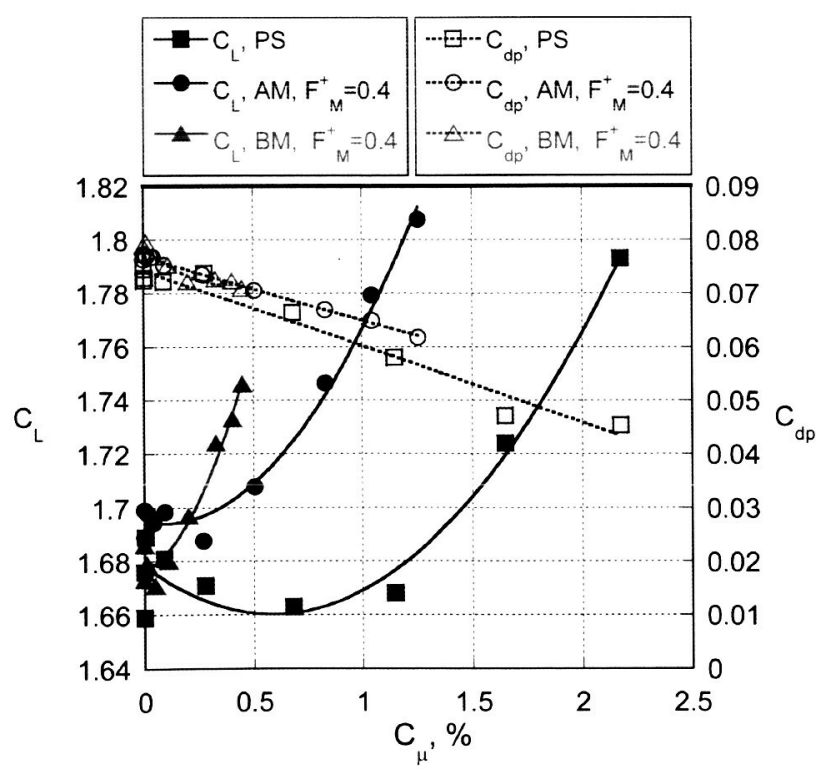

Figure 3. Comparison of Pure Sine, Amplitude and Burst Modulation excitation using the TE actuator, $R e_{c}=0.24 \times 10^{6}, \delta_{f}=20^{\circ}, \delta_{s}=-25^{\circ}, \alpha=6^{\circ}, F^{+}=$ $15.9, D c y, B M=0.25$. 
envelope.

Figure 4(b) presents $C_{L}$ and $C_{d p}$ plotted against Dcy for excitation from the TE actuator. The trends are slightly different from those generated by the flap actuator (Fig. 4(a)). Most significantly, $C_{d p}$ gradually decreases with increased Dcy and, $C_{L}$ continues to increase with increasing Dcy, but no additional lift increment is obtained using either slot with $D c y>50 \%$. It should also be noted that the lift response to the flap actuator is stronger and starts at a smaller Dcy than for the TE actuator.

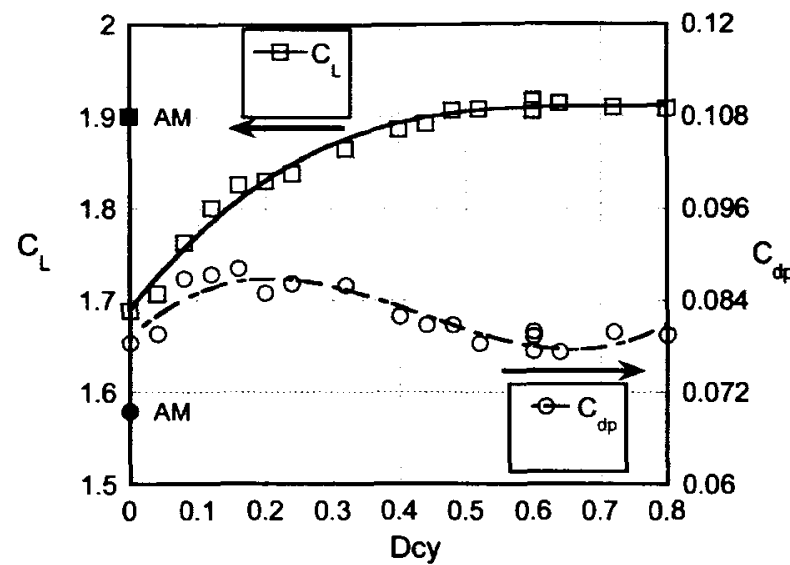

(a) Flap Slot \#3 Effect. $F^{+}=11.9, F_{M}^{+}=0.48$, $U_{p} / U_{\infty}=2.6$.

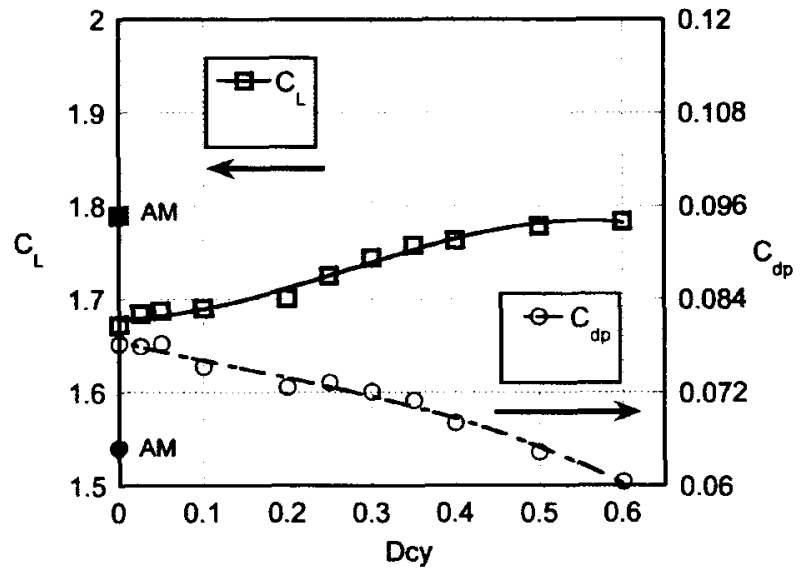

(b) TE actuator Effect. $F^{+}=15.6, F_{M}^{+}=0.39$, $U_{p} / U_{\infty}=3.3$.

Figure 4. Effect of Duty Cycle on TE Flap Region Actuation, $R e_{c}=0.24 x 10^{6}, \delta_{f}=20^{\circ}, \delta_{s}=-25^{\circ}, \alpha=6^{\circ}$.

In Fig. 5, the effect of $F_{M}^{+}$(based on the low frequency envelope signal) on $C_{L}$ and $C_{d p}$ for the TE and flap actuators is considered. The data show that the most effective $F_{M}^{+}$for the two actuators is $F_{M}^{+} \approx 0.5$, when lift increment is the purpose of the control scheme. The data also indicate that while both slot excitation locations increase $C_{L}$ in a similar manner, the TE actuator's excitation does a better job of decreasing the $C_{d p}$.

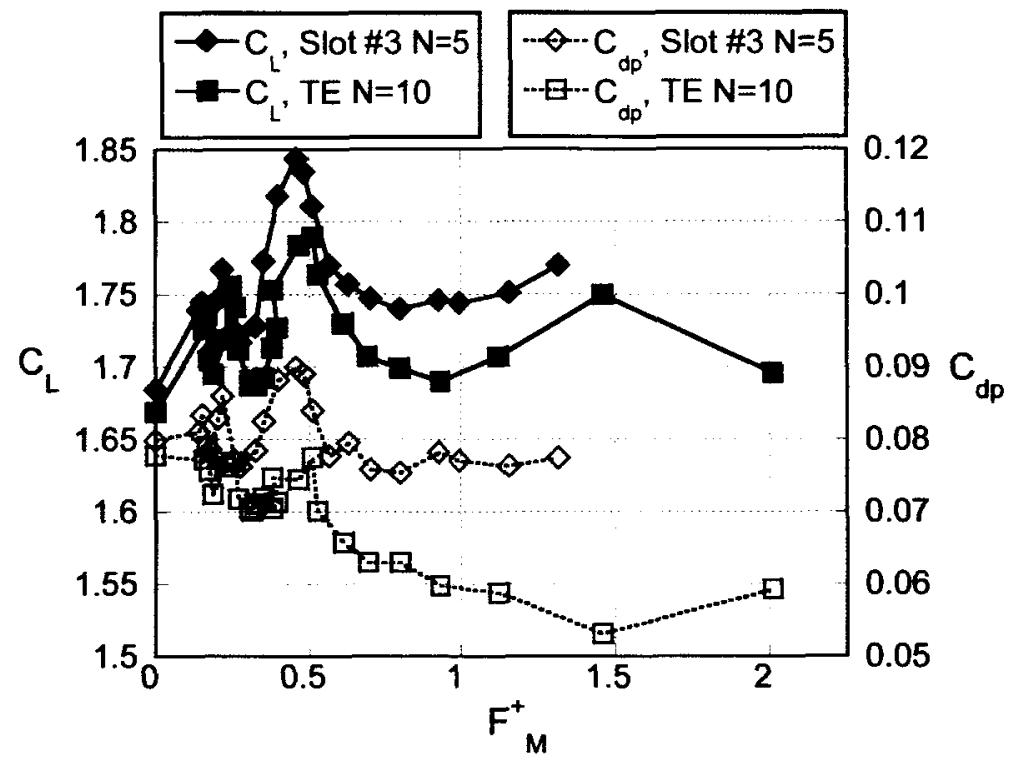

Figure 5. $C_{L}$ vs. $F_{M}^{+}$for the TE and Flap Slot \#3 actuators. $\delta_{f}=20^{\circ}, \delta_{s}=-25^{\circ}, \alpha=6^{\circ}, R e_{c}=0.24 x 10^{6}$ 


\section{Optimal Combination of TE and Flap Excitations}

The data presented in Section $\mathrm{A}$ indicate that $C_{L}$ can be increased by using either the TE actuator or the flap actuator. Furthermore, it was found that BM can be used to increase the momentum (and therefore the energy) efficiency of the actuation scheme. Following the (expected) finding that the same low frequency is effective for modulating the resonant frequency of both the TE and flap actuators, a common modulating waveform was used for the two resonant frequency based actuators so that the sensitivity to $F_{M}^{+}$phase between the two excitation output signals could be studied. The data were acquired at $R e_{c}=0.24 x 10^{6}, \delta_{s}=-25^{\circ}$, and $\delta_{f}=20^{\circ}$ using BM with Dcy $\approx 0.31$ and 0.26 for the TE and flap actuators, respectively, at several angles of attack. Figure 6(a) shows the dependence of $C_{L}$, at $\alpha=6^{\circ}$ and $12^{\circ}$, on the phase angle between the two excitations. Since one $f_{M}$ was used, $F_{M}^{+}$is slightly different for the two actuators. The data clearly indicate that the lift improvement $\left(C_{L, b a s}=1.68\right.$ and 2.16 for $\alpha=6^{\circ}$ and $\alpha=12^{\circ}$, respectively) is sensitive to the relative phase of the low frequency signal applied to the two actuators. The largest lift increment is obtained when the two actuators are operated with a phase shift between $\pm 30^{\circ}$ for both incidence angles presented. Similar data acquired at $\alpha^{\prime}$ 's between $0^{\circ}$ and $14^{\circ}$ and at higher $\delta_{f}$ 's indicate that, with the exception of the $\alpha=14^{\circ}$ case, the phase that provides the maximum increase in lift remains unchanged. When the separation criterion described in Ref. 9 is applied, the hot-film sensors on the model indicate that separation may be taking place, at least intermittently, upstream of the TE actuator slot at $\alpha=14^{\circ}$, thereby explaining the loss of phase sensitivity at $\alpha=14^{\circ}$.

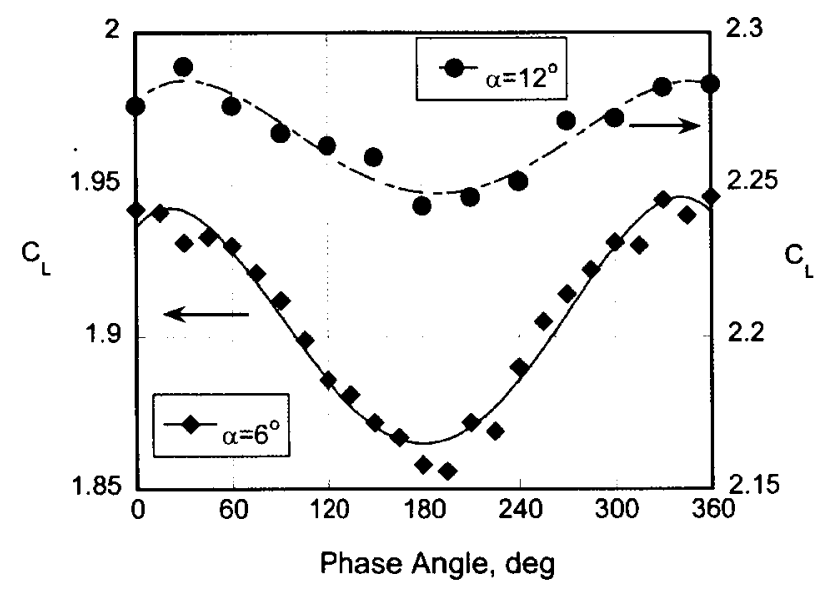

(a) $C_{L}$

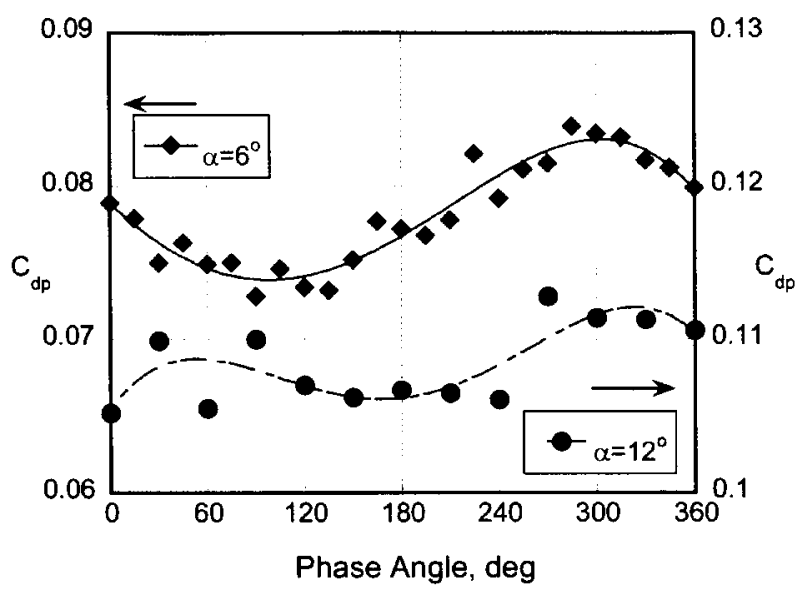

(b) $C_{d p}$

Figure 6. The effect of phase angle between flap slot \#3 and $\mathbf{T E}$ actuator on $C_{L}$ and $C_{d p} . F_{M, T E}^{+}=0.52, F_{M, s l o t 3}^{+}=$ $0.47, R e_{c}=0.24 \times 10^{6}, \delta_{s}=-25^{\circ}, \delta_{f}=20^{\circ}, f_{M}=42 \mathrm{~Hz}$.

In Fig. 6(b), the dependence of the form-drag, $C_{d p}$, on phase angle is presented for the $\alpha=6^{\circ}$ and $12^{\circ}$ cases. The phase angle that reduces $C_{d p}$ (below the baseline value of $C_{d p, b a s}=0.085$ and 0.14 for $\alpha=6^{\circ}$ and $\alpha=12^{\circ}$, respectively) most effectively is between $60^{\circ}$ and $150^{\circ}$. This phase shift does not coincide with the optimal phase for lift increment $\left( \pm 30^{\circ}\right)$ shown in Fig. 6(a). The dependence of $C_{d p}$ on phase is not as evident in the $\alpha=12^{\circ}$ data shown in Fig. 6(b). Data acquired at higher $R e_{c}$ and higher flap deflections with $\alpha=6^{\circ}$ show results similar to the $\alpha=6^{\circ}$ data of Fig. 6(a) and 6(b).

Mean pressure distributions relevant to the PIV data to be discussed later in this section are presented in Fig. 7. According to the pressure distributions, separation takes place between $\mathrm{x} / \mathrm{c}=0.8$ and $\mathrm{x} / \mathrm{c}=0.85$ for all cases considered. Therefore, significant performance variations do not take place due to delay of boundary layer separation. Alternatively, they occur due to an upstream effect caused by the vortices shed into the wake and the effect the modified wake and its vortices have on the flow upstream of the separation location, as will be shown at least partially by the PIV data. The only mode of excitation capable of meaningful separation delay is the flap slot \#3 actuator when operated alone. The in-phase combination of the two actuators operating in BM has both the strongest upstream and downstream effects as indicated by the most negative and positive pressures at $\mathrm{x} / \mathrm{c}=0.75$ and $\mathrm{x} / \mathrm{c}=1$, respectively.

In Fig. 8, the root mean square (rms) values of the short time fourier transforms (STFT) of the TE flap hot-films are presented. The use of this statistical analysis to determine the separation location is discussed 


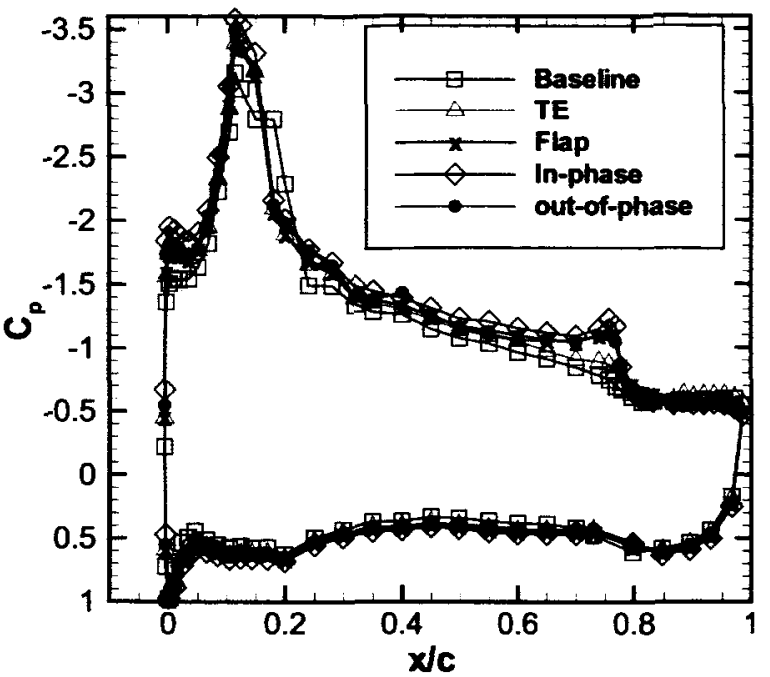

(a) Airfoil $C_{p}$

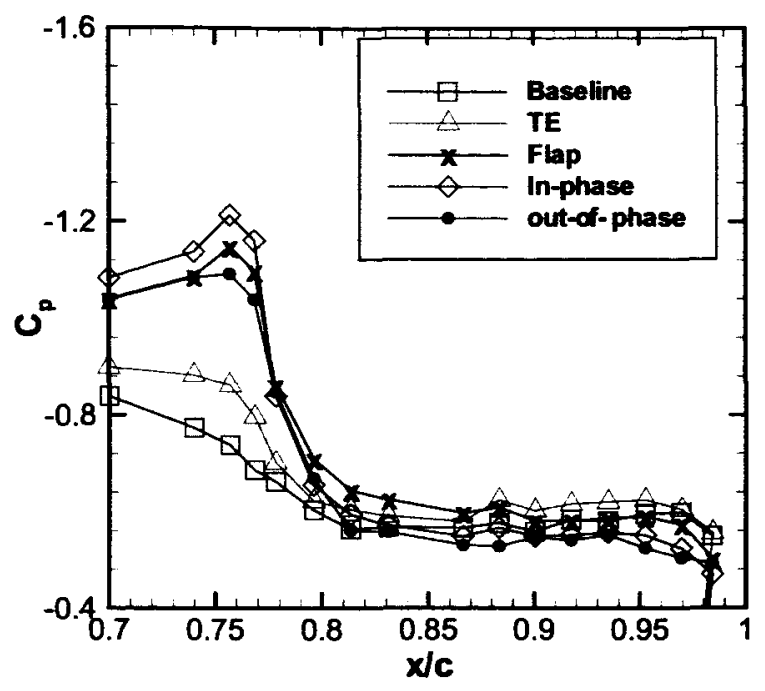

(b) Flap upper surface $C_{p}$

Figure 7. $C_{p}$ distributions comparing baseline with TE and flap slot\#3 excitation separately and in and out of phase. $F_{M, T E}^{+}=0.52, F_{M, \text { slot } 3}^{+}=0.47, R e_{c}=0.24 x 10^{6}, \delta_{s}=-25^{\circ}, \delta_{f}=20^{\circ}, f_{M}=40 \mathrm{~Hz}$.

in Ref. 9. Flow separation occurs when the rms of the STFT is greater than 0.225 , based on the validated criterion. The data indicate, in agreement with the $C_{p}$ distributions of Fig. 7, that flow separation on the TE flap is delayed by the different excitation modes used. Separation occurs between flap slot \#3 and the first hot-film on the flap, and it moves to $\mathrm{x} / \mathrm{c}=0.79$ with the TE excitation and to $\mathrm{x} / \mathrm{c}=0.866 \pm 0.005$ for the other excitations presented. The flap actuator is the most effective at delaying flow separation on the TE flap as noted previously. The peak rms value of the STFT is not captured for the flap excitation due to the hot-film sensor spacing. Since the largest increment in lift occurs when combining the TE and flap excitations in-phase, the lift increment has to be due to an upstream effect. This is due to the fixed separation point on the TE flap for the flap actuator and for the two actuators operating together.

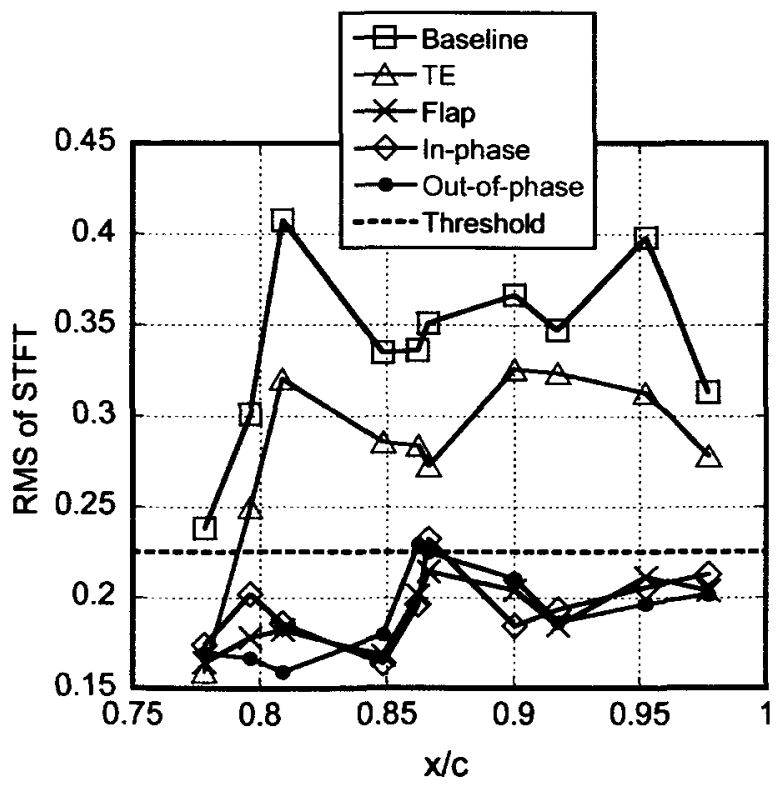

Figure 8. STFT RMS of TE Flap Hot-films for data of Figs. 7. $\delta_{f}=20^{\circ}, \delta_{s}=-25^{\circ}, \alpha=6^{\circ}, R e_{c}=0.24 \times 10^{6}$.
Figure 9 shows contours of mean (average of eight phases) $\mathrm{U}$ and $\mathrm{V}$ velocity components as well as vorticity for the baseline and the flow fields controlled by the TE actuator, the flap actuator and the TE and flap actuators combined with a phase angle of $0^{\circ}$ and $180^{\circ}$ between the $f_{M}=40 \mathrm{~Hz}$ of the two actuators. The baseline data of Figs. 9(a)-9(c) indicate, in agreement with the $C_{p}$ distributions of Fig. 7 and the STFT data of Fig. 8, that separation occurs just downstream of flap slot \#3. The TE excitation delays separation on the flap slightly based on the PIV data of Figs. 9(d)-9(f). The flap excitation and the combined flap and TE excitations seem to have similar effects on the TE flap flowfield based on the phase-averaged PIV data presented in Figs. 9(g)-9(o). The results agree with the PIV data and the STFT data that indicate flow separation is fixed on the TE flap for these cases. The flap excitation alone and combined in and out of phase with the TE excitation tend to bring the shear layer closer to the surface but do not keep the flow attached to the TE of the airfoil. The in-phase combination of the TE and flap excitations generates the largest velocity at the flap shoulder as indicated by the mean $U$ velocity data of Fig. 9(j) and the suction peak of Fig 7. 


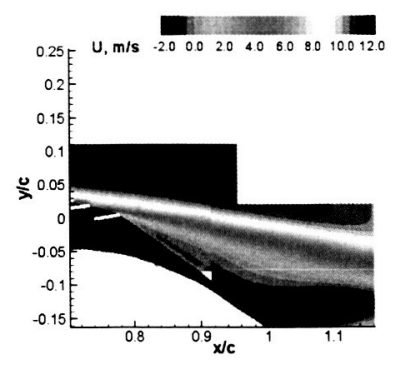

(a) Baseline Mean U

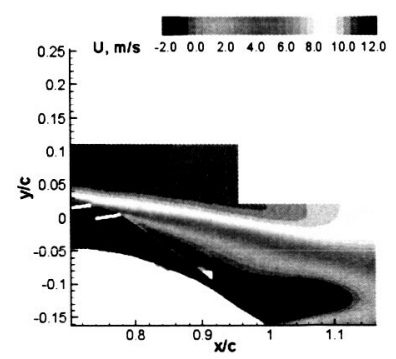

(d) TE U

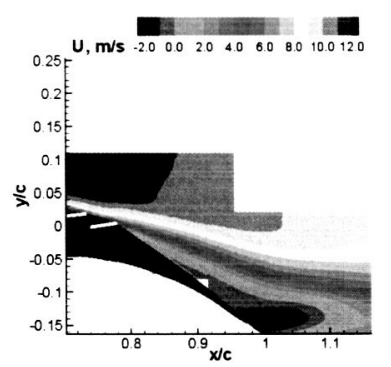

(g) Flap U

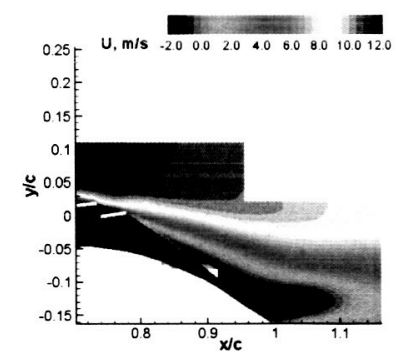

(j) In-phase U

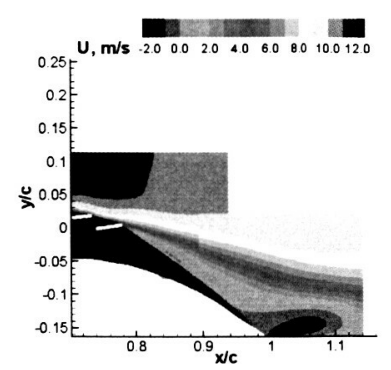

(m) Out-of-phase U

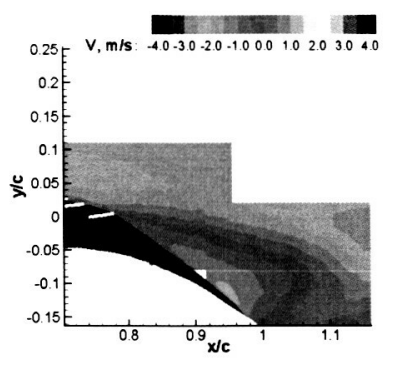

(b) Baseline Mean V

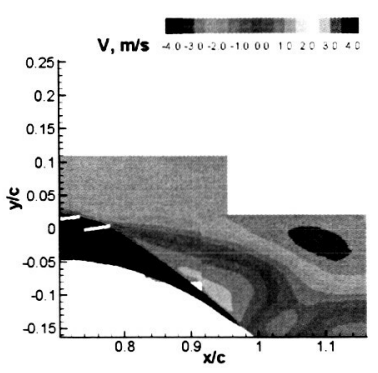

(e) TE V

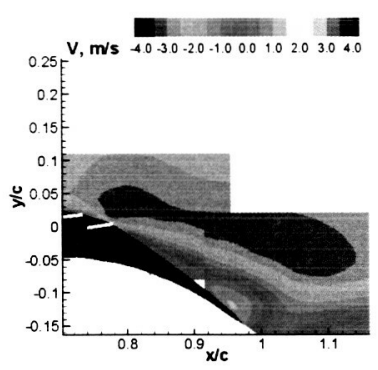

(h) Flap V

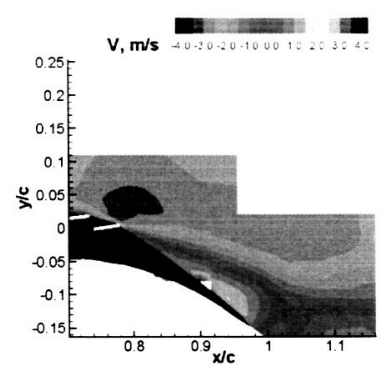

(k) In-phase V

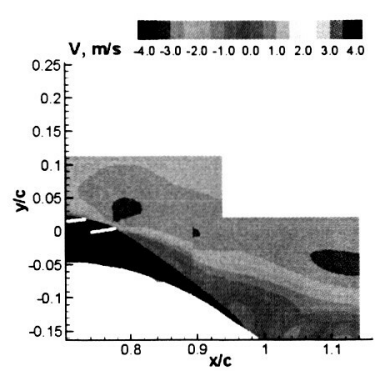

(n) Out-of-phase V

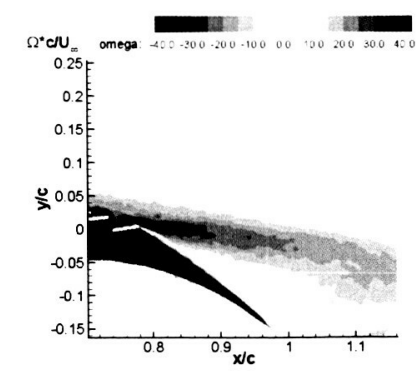

(c) Baseline Vorticity

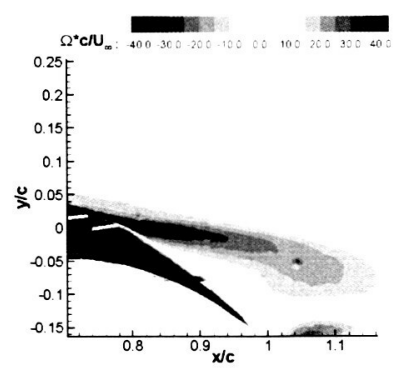

(f) TE Vorticity

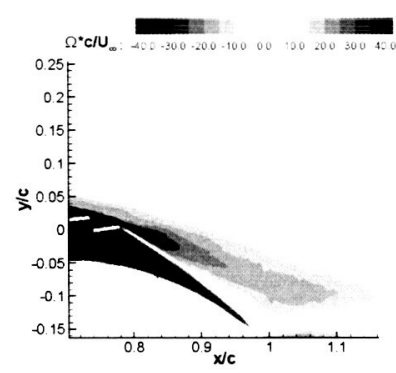

(i) Flap Vorticity

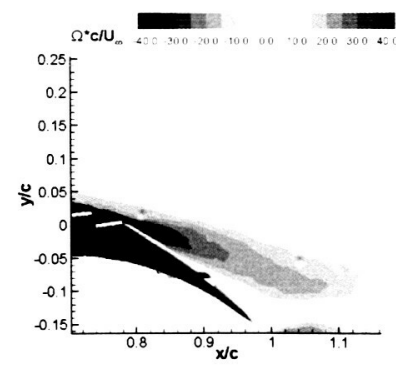

(1) In-phase Vorticity

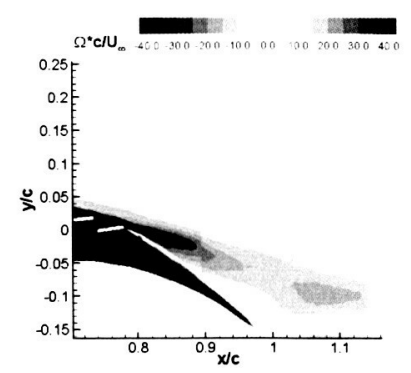

(o) Out-of-phase Vorticity

Figure 9. Phase-Averaged PIV data with TE and slot \#3 actuators at $R e_{c}=0.24 x 10^{6}, \alpha=6^{\circ}, \delta_{f}=-20^{\circ}, \delta_{s}=$ $-25^{\circ}, F_{M, T E}^{+}=0.5, F_{M 3}^{+}=0.45$ 
Figure 10 shows contours of phase-locked vorticity with velocity vectors overlayed, comparing the TE excitation, the flap excitation, and the in-phase and out-of-phase combinations of the TE and flap excitations. The TE excitation alone, even when operating at the optimal low frequency for lift increment, as shown in Figure 5, does not "lock" the upper surface "negative" vorticity to the excitation input. On the other hand, a very strong, compact, and coherent "positive" vortex is released from the lower surface of the airfoil TE at $\phi \approx 90^{\circ}$ and is convected only about $0.1 \mathrm{c}$ at $\sim 25 \mathrm{msec}$, resulting in normalized convection speed of $0.18 \mathrm{U}_{\infty}$. Even the mean "positive" vorticity, shown in Fig. 9(f), provides evidence of the averaged smeared positive vortex with increased magnitude compared to that of the baseline flow (Fig. 9(c)). The negative vorticity transported from the upper surface of the airfoil prior to separation at the flap shoulder that is increased by the excitation does not interact with the surface nor does it roll-up to form a discrete vortex prior to leaving the TE.

The modulated excitation emanating from flap slot \#3 is capable of rolling the upper surface boundary layer vorticity into discrete "negative" vortices that strongly interact with the TE flap upper surface before being shed into the wake. The "positive" vortex intermittently formed and shed from the lower surface of the TE flap seems to convect at the same streamwise velocity as that of the TE excitation but resides at a lower $\mathrm{y} / \mathrm{c}$, indicative of the larger lift generated by the flap excitation.

When both actuators operate in-phase, the "negative" vortex is formed closer to the slots (compared to the flap excitation alone), is characterized by larger negative vorticity that resides closer to the surface, and is convected slower downstream. The features of the "positive" vortex are similar to those of its flap (alone) excitation counterpart, with the minor exception of a slower streamwise convection velocity.

When both actuators operate out-of-phase, the negative vorticity rolls into a discrete vortex only after leaving the TE; therefore its effect on the flap performance is weaker.

\section{LE and TE Excitations Combined}

Figure 11 presents the effect of $F_{M}^{+}$(of the flap actuator) when the LE and flap actuator excitations are combined. Note that pure sine excitation was used for the LE actuator to eliminate relative phase and frequency sensitivity between the two actuators. The data indicate that the optimal flap $F_{M}^{+}$remains unchanged when the PS excitation of the LE is combined with the BM excitation of the flap at $\alpha=12^{\circ}$. Similar data acquired when combining the LE and TE actuators at $\alpha=12^{\circ}$ indicate that the addition of the PS, LE excitation tends to increase the optimal $F_{M}^{+}$of the TE actuator.

Figure 12 presents lift polars for various combinations of LE, TE, and flap actuator excitations. The data indicate that both the TE and flap actuators can be used to increase the effectiveness of the TE flap. The LE actuator increases the TE flap effectiveness somewhat, but has a larger effect on $C_{L, \max }$. When the excitations from all three actuators were combined, the improvements in performance below $C_{L, \max }$ were larger than the improvements seen when exciting the flow using the individual actuators. The improvement in $C_{L}$ using the three actuators was smaller near $C_{L, \max }$ than the combination of the LE and flap actuators (i.e., without the TE actuator). This combination (LE + FLP, Fig. 12) produced the greatest benefits near $C_{L, \text { max }}$, delaying stall by $1^{\circ}$ and increasing lift by about $16 \%$ at $\alpha=15^{\circ}$ as compared to the baseline at the same angle of attack and flap settings. $C_{L, \max }$ is increased by about $6 \%$.

Additional research is required to determine the reason for the reduced effect on $C_{L}$ when the three excitations are combined near $C_{L, \max }$.

\section{Summary and Conclusions}

The primary motivation of the present stage of the simplified high-lift system investigation was to combine several excitation sources. Excitation was introduced from an actuator placed upstream of the trailing edge (TE) flap shoulder to increase the momentum available for controlling flow separation at the TE flap shoulder at large TE flap deflections. The TE actuator was more efficient for lift increment when the high frequency excitation produced by the actuator at its resonant frequency was modulated at low frequency. The momentum required for a similar $C_{L}$ increment could be reduced by as much as $50 \%$ when using amplitude modulation (AM). The efficiency of the TE actuator could be further improved over that of the AM excitation by using burst modulation (BM) and varying the duty cycle.

Combining the TE and flap excitations increased the maximum lift over that produced by either actuator operating separately. The phase angle of the modulating waveform between the input signals had a significant 


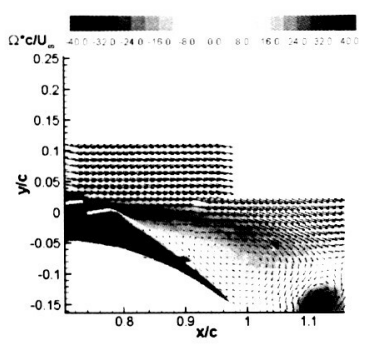

(a) $\phi=0^{\circ}$, TE Excitation

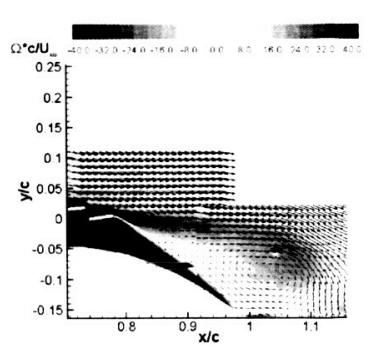

(e) $\phi=90^{\circ}$, TE Excitation

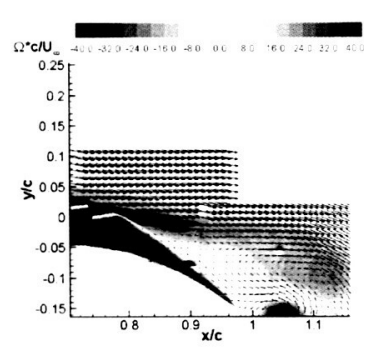

(i) $\phi=180^{\circ}$, TE Excitation

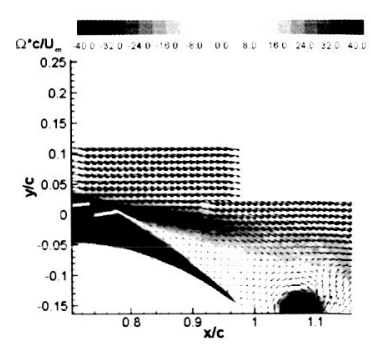

(m) $\phi=270^{\circ}$, TE Excitation

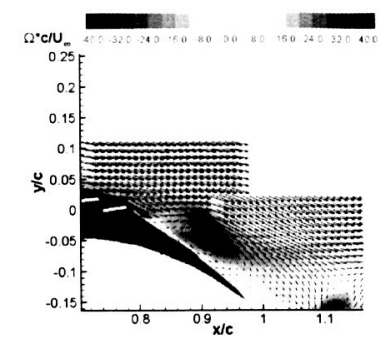

(b) $\phi=0^{\circ}$, Flap Excitation

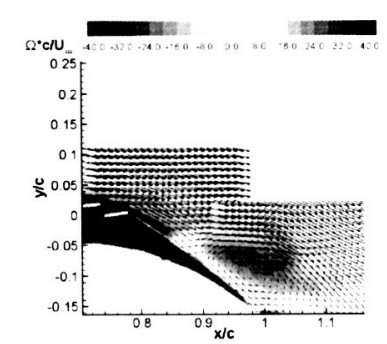

(f) $\phi=90^{\circ}$, Flap Excitation

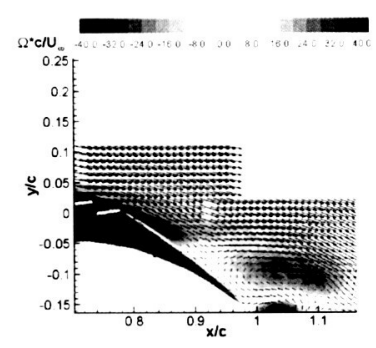

(j) $\phi=180^{\circ}$, Flap Excitation

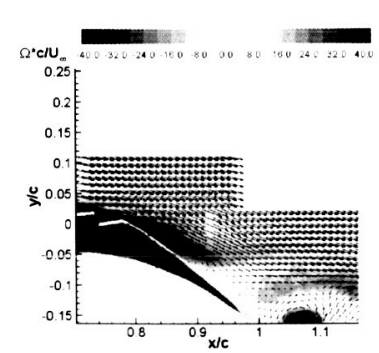

(n) $\phi=270^{\circ}$, Flap Excitation

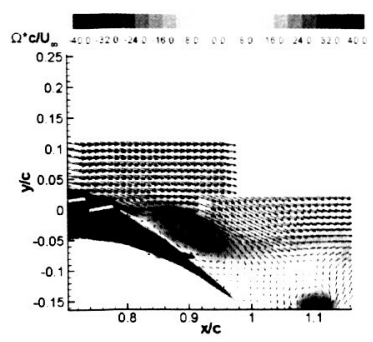

(c) $\phi=0^{\circ}, \mathbf{T E}+$ Flap InPhase Excitation

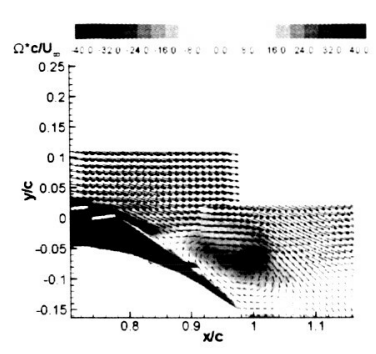

(g) $\phi=90^{\circ}$, TE + Flap InPhase Excitation

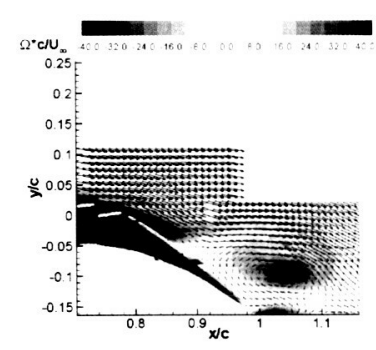

(k) $\phi=180^{\circ}$, TE +Flap In-Phase Excitation

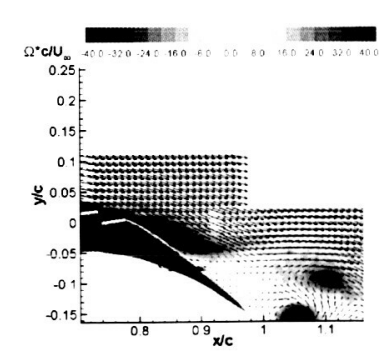

(o) $\phi=270^{\circ}, \mathbf{T E}+$ Flap In-Phase Excitation

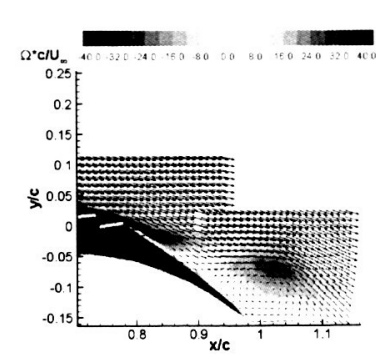

(d) $\phi=0^{\circ}, \mathrm{TE}+$ Flap Out-of-Phase Excitation

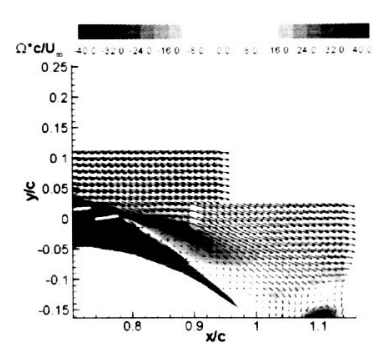

(h) $\phi=90^{\circ}, \quad$ TE +Flap Out-of-Phase Excitation

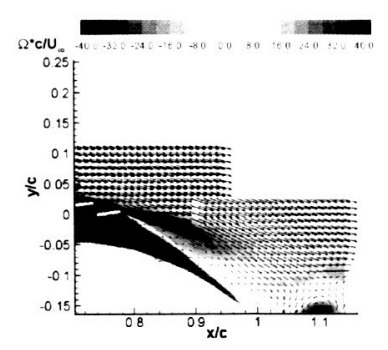

(1) $\phi=180^{\circ}$, TE +Flap Out-of-Phase Excitation

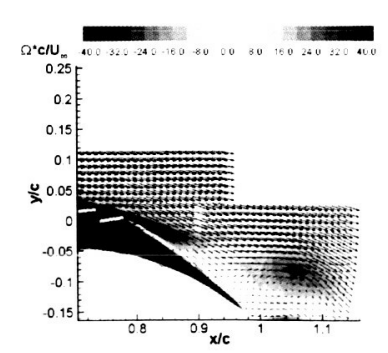

(p) $\phi=270^{\circ}, \mathbf{T E}+$ Flap Out-of-Phase Excitation

Figure 10. Phase-locked PIV data with TE and slot \#3 actuators at $R e_{c}=0.24 \times 10^{6}, \alpha=6^{\circ}, \delta_{f}=-20^{\circ}, \delta_{s}=$ $-25^{\circ}, F_{M, T E}^{+}=0.5, F_{M 3}^{+}=0.45$. Every 4 th vector in $\mathbf{x}$ and $\mathbf{y}$ displayed for clarity. 


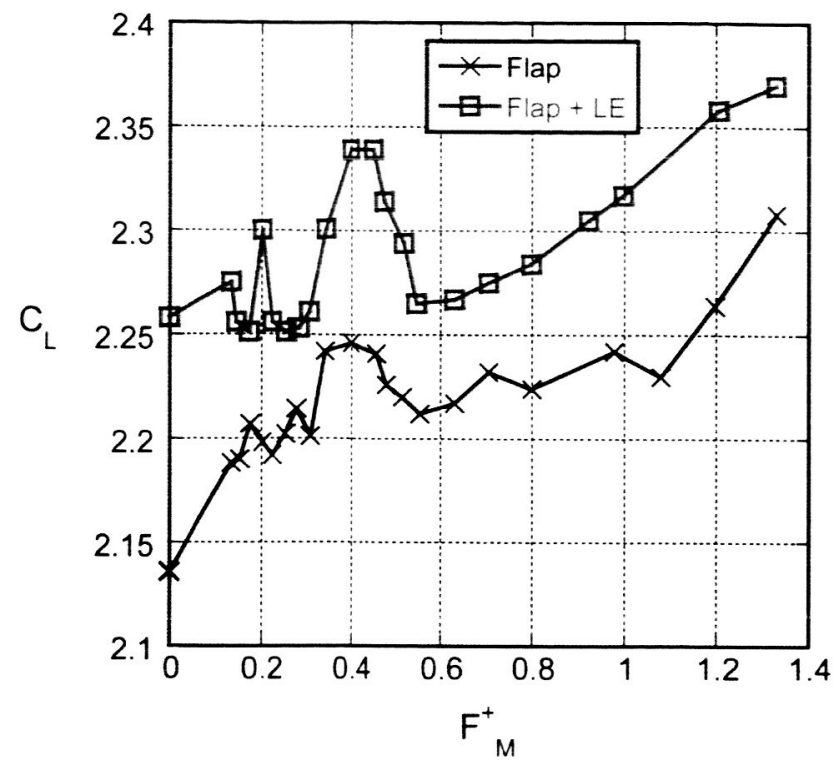

Figure 11. $C_{L}$ vs. $F_{M}^{+}$comparing Flap slot \#3 excitation and a combination of LE actuator and flap slot \#3 excitation. $R e_{C}=0.24 \times 10^{6}, \delta_{f}=20^{\circ}, \delta_{s}=25^{\circ}, \alpha=12^{\circ}$.

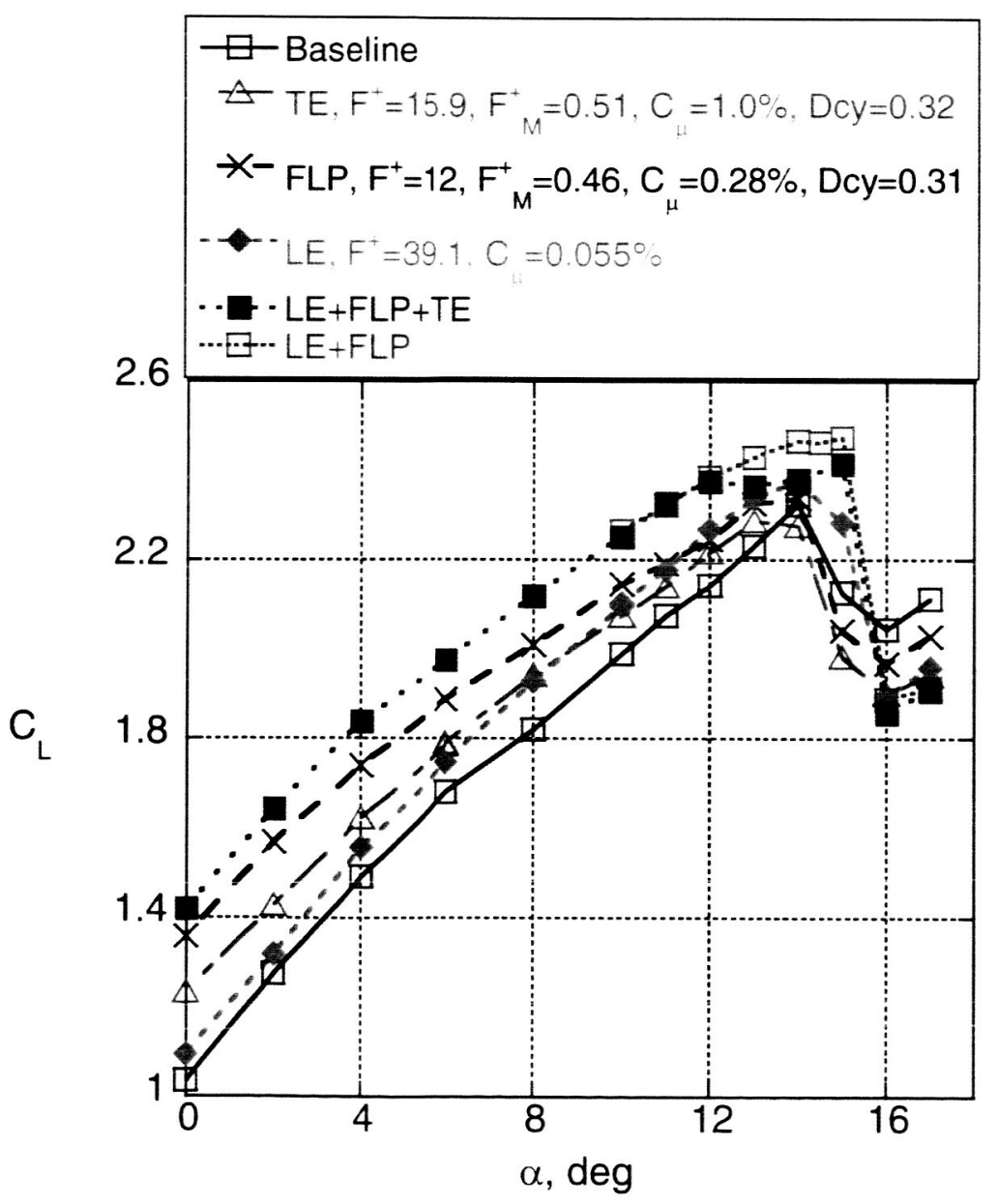

Figure 12. $C_{L}$ vs. a for various excitations. $R e_{c}=0.24 x 10^{6}, \delta_{f}=20^{\circ}, \delta_{s}=25^{\circ}$. 
but complex effect on both $C_{L}$ and $C_{d p}$ using BM. The maximum increment in lift occurred for a phase angle of $\pm 30^{\circ}$. The activation of the leading-edge-flap-shoulder actuator slightly increased the most effective excitation frequency at the trailing edge flap shoulder. The phase sensitivity, enabling enhanced performance due to a favorable interaction between two trailing edge flap actuators, disappeared near maximum lift incidence. The combined excitations from the LE and flap actuators produced the largest increment in lift near $C_{L, \text { max }}$, increasing $C_{L}$ by about $16 \%$ at an angle of attack of $15^{\circ}$ and $C_{L, \max }$ by $6 \%$. The maximum lift was increased by a two-stage mechanism. The leading edge actuator maintained attached flow to the trailing edge flap region. Activating the flap actuator in a manner that properly interacts with the vortex shedding process provided an additional lift increment. This interaction enhances the upstream suction while significantly enhancing vortex shedding downstream.

Additional research at higher $R e_{c}$ and in a wall-interference-free wind tunnel is required to interpret some of the complex behavior observed.

\section{Acknowledgements}

The authors would like to thank the following individuals for their support of the research program, Anna McGowan, William Sellers, Michael Walsh, Anthony Washburn, Luther Jenkins, John Lin, Norman Schaeffler, Richard White, George Hilton, Johnny Mau, Louis Hartzheim, Susan Palmer, and R. David Lewis.

\section{References}

${ }^{1}$ Seifert, A., Darabi, A., and Wygnanski, I., "On the Delay of Airfoil Stall by Periodic Excitation," Journal of Aircraft, Vol. 33, No. 4, 1996, pp. 691-699.

${ }^{2}$ Seifert, A. and Pack, L. G., "Oscillatory Control of Separation at High Reynolds Numbers," AIAA Journal, Vol. 37, No. 9, 1999, pp. 1062-1071.

${ }^{3}$ Seifert, A. and Pack, L. G., "Active Flow Separation Control on Wall-Mounted Hump at High Reynolds Numbers," $A I A A$ Journal, Vol. 40, No. 7, 2002, pp. 1363-1372.

${ }^{4}$ McClean, J. D., Crouch, J. D., Stoner, R. C., Sakurai, S., Feifel, G. E., Feifel, W. M., and Rush, H. M., "Study of the Application of Separation Control by Unsteady Excitation to Civil Transport Aircraft," NASA/CR 1999-209338, 1999.

${ }^{5}$ Lin, J. C. and Dominik, C. J., "Parametric Investigation of a High-Lift Airfoil at High Reynolds Numbers," Journal of Aircraft, Vol. 34, No. 4, 1997, pp. 485-491.

${ }^{6}$ G.V.Lachmann, Boundary Layer and Flow Control, Vol. 1, Pergamon Press Inc., 1961.

${ }^{7}$ Margalit, S., Greenblatt, D., Seifert, A., and Wygnanski, I., "Active Flow Control of a Delta Wing at High Incidence using Segmented Piezoelectric Actuators," AIAA Paper 2002-3270, June 2002, Accepted to Journal of Aircraft, March 2004.

${ }^{8}$ Pack, L. G., Schaeffler, N. W., Yao, C., and Seifert, A., "Active Control of Separation from the Slat Shoulder of a Supercritical Airfoil," AIAA Paper 2002-3156, June 2002.

${ }^{9}$ Melton, L. P., Yao, C., and Seifert, A., "Active Control of Separation from the Flap of a Supercritical Airfoil," AIAA Paper 2003-4005, June 2003.

${ }^{10}$ Naim, A., Greenblatt, D., Seifert, A., and Wygnanski, I., "Active Control of Cylinder Flow with and without a Splitter Plate using Piezoelectric Actuators," AIAA Paper 2002-3070, June 2002. 